\title{
Compensation model of multi-attribute decision making and its application to $\mathrm{N}$-version software choice
}

\author{
D.V. Gruzenkin ${ }^{1}$, G.V. Grishina ${ }^{1}$, M.S. Durmuş ${ }^{2}$, İ. Üstoğlu ${ }^{3}$, R.Yu.Tsarev ${ }^{1}$ \\ ${ }^{1}$ Siberian Federal University, Russia \\ gruzenkin.denis@good-look.su \\ ggv-09@inbox.ru \\ tsarev.sfuemail.ru \\ ${ }^{2}$ Pamukkale University, Turkey \\ msdurmus@pau.edu.tr \\ ${ }^{3}$ Yildiz Technical University, Turkey \\ ustoglu@yildiz.edu.tr
}

\begin{abstract}
Multi-attribute decision making deals with discrete finite set of alternatives. The solution to the multi-attribute decision making problem is the choice of an alternative from the set of all possible alternatives on the base of usually contradicting attributes. In this paper, a new multi-attribute decision making model is presented. The proposed model develops a linear compensatory process for the interconnected attributes. It concerns the overall ranking of the alternatives based on the attribute-wise ranking as well as the interaction and the combination of the attributes. The compensation model of multiattribute decision making is applied to $\mathrm{N}$-version software selection. $\mathrm{N}$-version programming is one of the well-known software development approach which ensures high dependability and fault tolerance of software. However, the problem of extra resource involvement arises since the N-version programming stipulates program redundancy. A set of characteristics/attributes have to be considered when choosing an optimal variant of $\mathrm{N}$-version software. The proposed compensation model of multi-attribute decision making provides a solution to this problem. Additionally, a case study on choosing N-version software for a real-life information and control system problem is provided to verify the correctness of our model.
\end{abstract}

Keywords: multi-attribute decision making, compensation model, dependability, $\mathrm{N}$-version software

\section{Introduction}

Modern control systems are characterized mainly by the ability of their data processing power where the control functions are executed by software. The main reason for this situation is that the control processes and the complex calculations of a huge amount of data cannot be carried out only by means of hardware [1]. 
The field of control systems application defines the requirements of the dependability of these systems [2]. There is a number of areas where the failure of control system can result in severe financial and economic losses or can harm human health and even cause death [3]. Since, the data processing and control is executed by means of software, then its dependability characteristics directly define the dependability of a control system as a whole [4].

One of the most well-established methodologies ensuring high level of dependability and fault tolerance of software is $\mathrm{N}$-version programming [5]. This methodology is based on the principle of program redundancy which allows to increase significantly the dependability of software for control systems [6, 7].

A large number of $\mathrm{N}$-version software modules, redundant versions, and also some restrictions of the real-world problems such as cost, execution time, memory requirements, dependability properties compose a decision making problem [7]. The problem is to determine the best variation of $\mathrm{N}$-version software for a control system by taking into account a number of criteria [8]. The solution to this problem can be obtained by applying a multi-attribute decision making method [9].

This paper considers the compensation model of multi-attribute decision making which allows to perform an overall alternative ranking based on the attribute-wise ranking as well as the interaction and the combination of the attributes. The proposed compensation model is applied to determine the best variation of $\mathrm{N}$-version software for a control system.

\section{Compensation model of multi-attribute decision making providing overall ranking alternatives on the base of attribute-wise ranking}

The compensation model of multi-attribute decision making suggests alternatives ranking according to their order of preference. The overall ranking of the alternatives is based on the attribute-wise ranking. The alternative that is assigned the first rank is the best one. Ranking of the alternatives by considering just the order of their preferences allows us to avoid scaling of the quality-type attributes. This process uses ordinal input data rather than cardinal ones $[9,10]$.

The model describes a linear compensatory process for the attribute interaction and combination. The overall ranking of the alternatives can be obtained by the attributewise ranking where the interaction of the attributes is ignored [9]:

$$
k_{i}=\sum_{j=1}^{n} k_{i j} ; i=1,2, \ldots, m,
$$

where

$$
\begin{aligned}
& n-\text { is the number of the attributes, } \\
& m-\text { is the number of the alternatives, } \\
& k-\text { is the number of ranks }(k=m), \\
& k_{i}-\text { is the overall rank of the alternative } i, \\
& k_{i j}-\text { is the rank of } i^{\text {th }} \text { alternative on the } j^{\text {th }} \text { attribute. }
\end{aligned}
$$


However, it is important to consider this dependence for the majority of decision making problems $[11,12,13]$. According to this, the compensation model of multiattribute decision making has been developed. In this case, the idea of compensation consists of accounting the interdependence between the attributes: the change of a value of one of them leads to the change of values of some other attributes.

Let us define the matrix $\pi$ as a square nonnegative matrix $m \times m$ where the element $\pi_{\mathrm{i} k}$ represents the number (or the frequency) of ranking of an alternative $A_{i}$ the $k^{\text {th }}$ attribute-wise ranking. The matrix $\pi$ is based on the matrix of the attribute-wise ranking $D$ of the alternatives.

$$
\begin{gathered}
\pi_{i j}=\sum_{l=1}^{n} I\left(D_{j l}^{i}\right) \cdot w_{l} ; i=1,2, \ldots, m ; j=1,2, \ldots, k, \\
I\left(D_{j l}^{i}\right)=\left\{\begin{array}{l}
1, \text { if } D_{j l}^{i}=i, \\
0, \text { if } D_{j l}^{i} \neq i,
\end{array}\right.
\end{gathered}
$$

where tribute $l$.

$$
I(x) \text { - is the indicator function; } \quad w_{l}-\text { is the weight coefficient of the at- }
$$

In the case of different weight coefficients the elements of the matrix $\pi$ represent the sum of attributes weights of the appropriate rank. The weight coefficients are supposed to be normed.

It is obvious that the element $\pi_{i k}$ defines the contribution of the alternative $A_{i}$ into the overall ranking. The more $\pi_{i k}$ value the more alternative $A_{i}$ deserves to be assigned to rank $k$.

Let us define a permutation matrix $Z$ as a square matrix $m \times m$ whose elements are $Z_{i k}=1$, if the alternative $A_{i}$ is assigned to the overall rank $k$, and $Z_{i k}=0$ otherwise. The objective function can be expressed as follows:

$$
\max _{Z_{i j}} \sum_{i=1}^{m} \sum_{j=1}^{k} \pi_{i j} Z_{i j}
$$

subject to:

$$
\begin{aligned}
& \sum_{j=1}^{k} Z_{i j}=1 ; i=1,2, \ldots, m, \\
& \sum_{i=1}^{m} Z_{i j}=1 ; j=1,2, \ldots, k .
\end{aligned}
$$

The conditions mean that the alternative $A_{i}$ can be assigned only to one rank, and the rank $k$ can be assigned to only one alternative.

The optimal permutation matrix representing the solution to the linear programming problem mentioned above is designated as $Z^{*}$. Then, the preference order can be achieved by multiplying the matrix $\pi$ by the matrix $Z^{*}$. 


\section{A case study on the application of the compensation model of multi-attribute decision making}

In this section, an application of the proposed compensation model of multi-attribute decision making is considered. In the first example the optimal solution is to be found out of three alternatives considering three attributes. The attribute-wise ranking of the alternatives is shown in Table 1.

Table 1. The attribute-wise ranking of the alternatives

\begin{tabular}{ccccc}
\hline \multicolumn{2}{c}{ Attribute } & $X_{1}$ & $X_{2}$ & $X_{3}$ \\
\hline \multirow{2}{*}{} & $A_{1}$ & $A_{1}$ & $A_{2}$ \\
2 & $A_{2}$ & $A_{3}$ & $A_{1}$ \\
& $A_{3}$ & $A_{2}$ & $A_{3}$ \\
\hline
\end{tabular}

The attribute-wise ranking of the attributes is represented by the matrix $D$. The indexes of the alternatives shown in Table 1 are considered to be the elements of the matrix $D$. Therefore, the matrix $D$ can be written as follows:

$$
D=\left[\begin{array}{lll}
1 & 1 & 2 \\
2 & 3 & 1 \\
3 & 2 & 3
\end{array}\right]
$$

The matrix $\pi$ can be developed on the basis of the matrix $D$ whose elements are presented by the number of alternative assignments of each rank. The first alternative is assigned to the first rank twice, the second rank once, and the first alternative is not assigned the third rank. The first line of the matrix $\pi$ reflects the first alternative assignments:

$$
\pi=\left[\begin{array}{lll}
2 & 1 & 0 \\
1 & 1 & 1 \\
0 & 1 & 2
\end{array}\right]
$$

Applying the attributes weight coefficients $w_{1}=0.2, w_{2}=0.4, w_{3}=0.4$, the elements of the matrix $\pi$ can be rewritten as follows:

$$
\pi=\left[\begin{array}{ccc}
0.2+0.4 & 0.4 & 0 \\
0.4 & 0.2 & 0.4 \\
0 & 0.4 & 0.2+0.4
\end{array}\right]=\left[\begin{array}{ccc}
0.6 & 0.4 & 0 \\
0.4 & 0.2 & 0.4 \\
0 & 0.4 & 0.6
\end{array}\right]
$$

The optimal permutation matrix $Z^{*}$ is as follows:

$$
Z^{*}=\left[\begin{array}{lll}
1 & 0 & 0 \\
0 & 1 & 0 \\
0 & 0 & 1
\end{array}\right] .
$$

The most left-top element of matrix $Z^{*}$ reflects assignment of the first alternative (the first column) to the first rank (the first line). The preference order is obtained by multiplying the matrix $\pi$ by the matrix $Z^{*}$ is as follows:

$$
A_{1} \succ A_{2} \succ A_{3} \text {. }
$$


Thus, the best alternative is alternative $A_{1}$.

Let us consider another example. The values of the attributes of three alternatives are shown in Table 2.

Table 2. The values of the attributes

\begin{tabular}{ccccc}
\hline Alternative & Cost $(\$)$ & Reliability & Processor time $(\mathrm{msec})$ & RAM volume $(\mathrm{Gb})$ \\
\hline$A_{1}$ & 1000 & 0.90 & 7 & 2 \\
$A_{2}$ & 1500 & 0.95 & 5 & 4 \\
$A_{3}$ & 2000 & 0.99 & 2 & 8 \\
\hline
\end{tabular}

The attribute-wise ranking of the alternatives is shown in Table 3.

Table 3. Attribute-wise ranking of the alternatives

\begin{tabular}{ccccc}
\hline Rank & Cost $(\$)$ & Reliability & Processor time $(\mathrm{msec})$ & RAM volume $(\mathrm{Gb})$ \\
\hline 1 & $A_{1}$ & $A_{3}$ & $A_{3}$ & $A_{1}$ \\
2 & $A_{2}$ & $A_{2}$ & $A_{2}$ & $A_{2}$ \\
3 & $A_{3}$ & $A_{1}$ & $A_{1}$ & $A_{3}$ \\
\hline
\end{tabular}

The overall rank can be obtained on the basis of the attribute-wise ranking (1):

$k_{1}=1+3+3+1=8$,

$k_{2}=2+2+2+2=8$,

$k_{3}=3+1+1+3=8$,

where $k_{i}$ is a rank of the alternative $A_{i}$.

The result provides a decision maker with no information.

Applying the proposed compensation model of multi-attribute decision making the matrix $\pi$ can be obtained as follows:

$$
\pi=\left[\begin{array}{ccc}
0.5 & 0 & 0.5 \\
0 & 1 & 0 \\
0.5 & 0 & 0.5
\end{array}\right] .
$$

In this case, two instances of the matrix $Z^{*}$ are relevant:

$$
Z_{1}^{*}=\left[\begin{array}{ccc}
0 & 0 & 1 \\
0 & 1 & 0 \\
1 & 0 & 0
\end{array}\right] \text { and } Z_{2}^{*}=\left[\begin{array}{ccc}
1 & 0 & 0 \\
0 & 1 & 0 \\
0 & 0 & 1
\end{array}\right],
$$

The compensation model demonstrates that the alternatives $A_{1}$ and $A_{3}$ are more preferable than the alternative $A_{2}$.

Let us define the weight coefficients reflecting the importance of attributes:

$$
\begin{aligned}
& \omega_{\text {cost }}=0.2 \\
& \omega_{\text {rel }}=0.5 \\
& \omega_{\text {time }}=0.1
\end{aligned}
$$


$\omega_{\text {vol }}=0.2$

Then matrixes $\pi$ and $Z^{*}$ are as follows:

$$
\begin{gathered}
\pi=\left[\begin{array}{ccc}
0.4 & 0 & 0.6 \\
0 & 1 & 0 \\
0.6 & 0 & 0.4
\end{array}\right], \\
Z^{*}=\left[\begin{array}{lll}
0 & 0 & 1 \\
0 & 1 & 0 \\
1 & 0 & 0
\end{array}\right], \\
A_{3} \succ A_{2} \succ A_{1} .
\end{gathered}
$$

In this case, the most preferable alternative is the alternative $A_{3}$.

\section{Choice of the $\mathrm{N}$-version software for an information and control system}

The compensation model of the multi-attribute decision making model was applied to solve a real-life problem for the choice of $\mathrm{N}$-version software for an information and control system used in a Krasnoyarsk transport company.

An analysis of the existing information and control system of the company was performed prior to the modernization of its software. The company provides a user with a workplace equipped with a computer which continuous functioning was supported by an uninterruptible power supply (UPS).

The core of the system is a server with an individual UPS of extended capacity and some network equipment. Besides, the information and control system includes a rented virtual server which is placed remotely on the Internet. In case of the primary server failure, the requests from clients are redirected to this backup server. Interacting with controllers, both of these servers increase external data traffic doubling network load. As a result, it negatively affects the productivity of the whole information and control system.

Only the primary server interacts with controllers. At the same time, it exchanges data with a backup server which continuously updates its status. If data exchange with the primary server stops, the backup server supposes that the primary server has failed and takes over its functions. After the failure of the primary server is fixed, it restores and switches on again. The primary server reads the current state from the backup server and retakes over its functions as a primary server.

The company's information and control system can be considered rather unreliable as data duplication occurs for the data transferred on a network, i.e. server failure will result in malfunctioning of the regulated subsystems. Thus, for instance, the occurrence of a failure in at least one transmission channel of the system will lead to an inappropriate control of the operations execution and can result in essential financial losses. 
The increase of the dependability and implementation of the fault tolerance of software of the information and control system is applied on programming level according to the N-version methodology. There is a set of possible variants for $\mathrm{N}$ version software to implement. The problem is to choose an optimal alternative that is a variant of $\mathrm{N}$-version software for applying to the information and control system. The solution to this multi-attribute decision making problem is to be obtained on the basis of the attribute values. The attribute values are shown in Table 4.

Table 4. Variants of the N-version software

\begin{tabular}{cccccc}
\hline Attribute & Cost & Time & Vol & Reliability & MTTF \\
\hline & 11000 & 850 & 15 & 0.99911 & 5.11 \\
$A_{1}$ & 12000 & 400 & 11 & 0.99934 & 3.89 \\
$A_{2}$ & 14000 & 400 & 15 & 0.99956 & 4.65 \\
$A_{3}$ & 14000 & 300 & 14 & 0.99921 & 4.35 \\
. & 15000 & 700 & 12 & 0.99965 & 3.77 \\
$A_{4}$ & 18000 & 500 & 10 & 0.99943 & 5.34 \\
$A_{5}$ & 19000 & 600 & 7 & 0.99932 & 4.54 \\
$A_{6}$ & 21000 & 500 & 11 & 0.99984 & 3.56 \\
$A_{8}$ & 24000 & 300 & 9 & 0.99977 & 5.66 \\
\hline
\end{tabular}

Notation:

Cost - Cost of the N-version software, \$

Time - Processor time, sec.

Vol - Volume of RAM required, Gb

Reliability - Reliability of the N-version software

$M T T F$ - Mean time to failure, months

The importance of an attribute is reflected by its weight:

$$
\begin{aligned}
& \omega_{\text {Cost }}=0.20 ; \\
& \omega_{\text {Time }}=0.15 ; \\
& \omega_{\text {Vol }}=0.10 ; \\
& \omega_{\text {Reliability }}=0.30 ; \\
& \omega_{\text {MTTF }}=0.25 .
\end{aligned}
$$

The result of the calculations according to the proposed compensation model of multi-attribute decision making is as follows:

$$
A_{8} \succ A_{9} \succ A_{2} \succ A_{3} \succ A_{6} \succ A_{4} \succ A_{7} \succ A_{5} \succ A_{1}
$$

Thus, the optimal solution to the given multi-attribute decision making problem is the alternative $A_{8}$, that represents a certain variants of the N-version software implementation.

The reliability of the chosen $\mathrm{N}$-version software for the information and control system is $99,984 \%$ that is $0,72 \%$ more than the previous value of reliability. The eco- 
nomic effect from modernization of the information and control system software was assessed by means of Advisor Client \& Server Model $[14,15]$. The results of economic assessment show that even with some extra modification costs the total expenses in case of system failure are reduced for $\$ 27.981,35$.

\section{Conclusion}

Redundant software such as $\mathrm{N}$-version software requires more resources than classic one-version software. Therefore, software designers and developers face the problem of compromise between benefits and losses. To increase the dependability of the software and avoid extra expenses at the same time, they have to deal with multiattribute decision making. The proposed compensation model in this paper allows to solve this multi-attribute decision making problem.

In this paper we present the results of an application of the compensation model of multi-attribute decision making to choose the optimal variant of the $\mathrm{N}$-version software for an information and control system. The results demonstrate the ability of the proposed model to bring us to the solution to the given multi-attribute decision making problem.

The compensation model of the multi-attribute decision making allows to perform the overall ranking of the alternatives in the order of their preferences. The overall ranking is based on the attribute-wise ranking. The proposed model takes into account the interaction and the combination of the attributes. The compensation model concerns ordinal preferences rather than cardinal ones. It allows to avoid problems related to the scaling of the attribute values and make it possible to consider both quantityand quality-type attributes. The only input data for the model is the attribute-wise ranking of the alternatives. The compensation model of multi-attribute decision making describes a linear compensatory process. Therefore, the solution can be obtained by means of an available mathematical package.

The proposed multi-attribute decision making model was also applied to solve a real-life problem to verify its correctness. The optimal variant of the $\mathrm{N}$-version software has been selected with assistance of the model.

Moreover, the developed compensation model of the multi-attribute decision making can be applied to solve a decision making problem in the discrete finite space of alternatives where the decision is based on the attribute values of the alternatives.

\section{References}

1. Tsai Wei-Tek, Zhou Xinyu, Paul Raymond A., Chen Yinong, Bai Xiaoying: A Coverage Relationship Model for Test Case Selection and Ranking for Multi-version Software. High Assurance Services Computing, pp. 285-311. (2009)

2. Eckhardt, D.E., Lee, L.D.: A Theoretical Basis for the Analysis of Multiversion Software Subject to Coincident Errors. IEEE Transactions on Software Engineering, pp. 1511-1517. (1985) 
3. Ping Guo, Xin Liu, Qian Yin: Methodology for Reliability Evaluation of N-Version Programming Software Fault Tolerance System. In Proc. IEEE Computer Science and Software Engineering International Conference, pp. 654-657. (2008).

4. Zuzana, K.: Software Reliability Models. In Proc. IEEE Radioelektronika, 2007 17th International Conference. (2007)

5. Avizienis, A., Chen, L.: On the implementation of $\mathrm{N}$-version programming for software fault-tolerance during program execution. In Proc. of IEEE Computer Society International Conference on Computers, Software and Applications Conference, COMPSAC, pp. 149155. (1977)

6. Gruzenkin, D.V., Tsarev, R.Y., Pupkov, A.N.: Technique of selecting multiversion software system structure with minimum simultaneous module version usage. Advances in Intelligent Systems and Computing, vol. 465, pp. 375-386. (2016)

7. Kulyagin, V.A., Tsarev, R.Yu., Prokopenko, A.V., Nikiforov, A.Yu., Kovalev, I.V.: Nversion design of fault-tolerant control software for communications satellite system. In: International Siberian Conference on Control and Communications (SIBCON), pp. 1-5. (2015)

8. Sklyar, V., Karchenko, V.: A Method of Multiversion Technologies Choice on Development of Fault-Tolerant Software Systems. Workshop on Methods, Models and Tools for Fault Tolerance, pp. 148-157. (2007).

9. Hwang Ching-Lai, Yoon Kwangsun: Methods for Multiple Attribute Decision Making. Multiple Attribute Decision Making, pp 58-191. (1981)

10. Bernardo, J.J., Blim, J.M.: A programming model of consumer choice among multiattributed Brands. Journal of Consumer Research, vol. 4, pp. 111-118. (1977)

11. Saidi Mehrabad, M., Fathian Brojeny, M.: The Development of an Expert System for Effective Selection and Appointment of the Jobs Applicants In Human Resource Management. Computers \& Industrial Engineering, 53(2), 306-312. (2007)

12. Gülfem Işıklar, Gülçin Büyüközkan: Using a multi-criteria decision making approach to evaluate mobile phone alternatives. Computer Standards \& Interfaces, pp. 265-274. (2007)

13. Xu, Zeshui: Uncertain Multi-Attribute Decision Making. Methods and Applications. Springer Heidelberg New York Dordrecht London (2015)

14. Schwalbe, Kathy: Information Technology Project Management. Cengage Learning: Australia, Brazil, Mexico, Singapore, United Kingdom, United States (2015)

15. Hila Chalutz Ben - Gal, Shay S. Tzafrir: Consultant-client relationship: one of the secrets to effective organizational change. Journal of Organizational Change Management, Vol. 24, Issue 5, pp. 662 - 679. (2011) 